\title{
Linx
}

Revue des linguistes de l'université Paris X Nanterre

$57 \mid 2007$

Études de syntaxe : français parlé, français hors de France, créoles

\section{L'interrogation dans un corpus de français parlé en Acadie. Formes de la question et visées de l'interrogation}

\section{Laurence Arrighi}

\section{OpenEdition}

Journals

Édition électronique

URL : http://journals.openedition.org/linx/277

DOI : $10.4000 /$ linx.277

ISSN : 2118-9692

Éditeur

Presses universitaires de Paris Nanterre

Édition imprimée

Date de publication : 1 décembre 2007

Pagination : 47-56

ISSN : 0246-8743

Référence électronique

Laurence Arrighi, «L'interrogation dans un corpus de français parlé en Acadie. Formes de la question et visées de l'interrogation », Linx [En ligne], 57 | 2007, mis en ligne le 15 février 2011, consulté le 15 mai 2020. URL : http://journals.openedition.org/linx/277 ; DOI : https://doi.org/10.4000/linx.277 


\title{
L'interrogation dans un corpus de français parlé en Acadie Formes de la question et visées de l'interrogation
}

\author{
Laurence Arrighi \\ Université de Moncton
}

Comme pour d'autres variétés de français, il y a peu d'études des structures interrogatives en français acadien. Beaucoup de corpus sont, en effet, des interviews, qui ne favorisent pas ces structures. Mon corpus de français parlé en Acadie ${ }^{1}$ est constitué d'enregistrements de discours ordinaires, notamment de discussions lors de repas de famille ou de conversations amicales, et d'enregistrements effectués par des Acadiens dans leur famille ou leur cercle d'amis. Ces sources présentent beaucoup d'interrogatives.

Outre la relative rareté des études consacrées à l'interrogation en langue parlée, les modalités de l'interrogation m'ont paru aussi prometteuses pour la multiplicité des formes possibles, et pour proposer une autre approche face au regard sociologique

\footnotetext{
${ }^{1}$ Ce corpus est constitué par l'enregistrement de 37 personnes d'âge et de milieu différents, effectué en 2002-2003 en divers lieux de la francophonie acadienne. Ces données orales ont été enrichies de données écrites, provenant du courrier des lecteurs du quotidien L'Acadie Nouvelle (énoncés notés AN). Pour des informations supplémentaires sur le corpus, voir Arrighi, 2005.
} 
(sociolinguistique au sens étroit ${ }^{2}$ ) souvent jeté sur cette pluralité de formes. Plus précisément, je m'éloigne ici des analyses qui discriminent les formes interrogatives à partir de la notion de registre de langue, pour y substituer une approche pragmatique.

\section{Pour situer cette étude}

\subsection{Quelques caractéristiques sociolinguistiques du français en Acadie}

Ce n'est pas une frontière politique constituée qui fonde aujourd'hui l'Acadie, mais l'existence de communautés de langue française dispersées dans les trois provinces maritimes du Canada (Nouveau-Brunswick, Ile du Prince Edouard, Nouvelle-Ecosse). Pour des raisons historiques et en dépit d'acquis législatifs en matière de langue obtenus à la fin des années 1960, la situation reste largement diglossique. L'anglais est dominant partout, en témoigne un bilinguisme fort des locuteurs de langue maternelle française. Au Nouveau-Brunswick, parce que le nombre le permet et surtout parce que la société acadienne s'est prise en main, cette dernière a pu se doter d'institutions sur le plan linguistique, et aujourd'hui l'égalité est atteinte dans certains secteurs ${ }^{3}$.

Le français acadien oscille sur un continuum complexe, du plus ou moins traditionnel au plus ou moins anglicisé, au plus ou moins standardisé.

\subsection{Motivation de l'étude et choix d'observation}

En français parlé, l'interrogation montre une grande variabilité. Au dépouillement du corpus, il apparaît que toutes les possibilités recensées pour interroger sont attestées: intonation, inversion, particules tu ou ti postposées au verbe, mots du discours (là, ben, pis ou encore hein), terme interrogatif seul ou non. Ces procédés peuvent se combiner. Pour cette étude, je laisserai de côté les procédés prosodiques et lexico-pragmatiques, comme les particules postposées au verbe et les mots du discours. Je me concentrerai sur les procédés morphosyntaxiques (inversion et terme introducteur), étant entendu qu'il ne s'agit que d'une partie d'une riche panoplie. Ce dernier type de constructions se trouve essentiellement dans les interrogations totales ou partielles portant sur les circonstances ou l'objet. C'est le cas pour la majorité des témoins, avec de grandes différences.

Devant la question : pourquoi le locuteur utilise-t-il une forme plutôt qu'une autre, j'écarte l'hypothèse d'une corrélation entre structures interrogatives et caractéristiques sociales du locuteur, qui établirait des liens univoques au niveau d'études, à la profession, ou aux «facteurs sociodémographiques et culturels » (Barbarie, 1982). Ces associations, en effet, ne permettent pas de comprendre pourquoi un même locuteur alterne différentes formes dans une même situation de communication. C'est pour

\footnotetext{
2 J'entends par là une perspective corrélative, dans laquelle l'utilisation de telles ou telles formes linguistiques est expliquée par le recours au profil social du locuteur.

${ }^{3}$ Le français et l'anglais sont langues co-officielles de cette province depuis 1969, statut qui garantit un usage institutionnel relativement équilibré des deux langues. En revanche, dans l'usage courant, on note encore un assez grand déséquilibre au profit de l'anglais.
} 
cette raison qu'il ne sera pas fait mention du profil sociologique des locuteurs dont je cite les productions. Une autre raison, théorique, pousse à laisser de côté la démarche corrélative : c'est qu'elle présume une correspondance sémantique et pragmatique entre les formulations, qui est loin d'être établie (Blanche-Benveniste, 1997, Coveney, 1997, Gadet, 1997b).

En regard des données recueillies et de leur comparaison avec des études d'autres variétés orales, il apparait plus fructueux d'interroger la notion de variabilité en termes de tendances selon la visée de la question. Je me concentrerai sur la question visant à obtenir une information (demande de dire). Le rapport de la forme de l'interrogation aux visées pragmatiques a été fait par Coveney $(1995,1996,1997)$, ou Quillard (2001) pour des corpus de France, et il est suggéré pour tous les champs de la variation en syntaxe (Gadet, 1997b). Mais, à ma connaissance, il n’a pas été appliqué aux interrogatives en français parlé hors de France, du moins pour les variétés nordaméricaines. Cela me semble à mettre au compte de postures de recherche reliant variétés diatopique et diastratique, et sous-entendant ainsi que le maximum de divergence par rapport à la norme se rencontre auprès des couches les moins éduquées. De même, bien des grammaires du français qualifient certaines des formes que j'étudierai de «familière », " très familière », " populaire », sans bien définir ce qu'elles entendent par là. L'hypothèse qui oppose les formes selon le niveau de langue (on dit la même chose dans un autre registre) ou les usages sociaux semble «inhiber» (Gadet, 1997b, p. 8) d'autres hypothèses, telles que la recherche de nuances sémantiques ou pragmatiques, ou de contraintes syntaxiques.

\subsection{Démarches d'observation et modalités d'analyse}

Je présente ici un relevé des modalités de l'interrogation qui vise à mettre au jour des tendances, et se limite à deux types de questions (deux visées de la question) dans la demande de dire : celle formulée pour obtenir une réponse affirmative ou négative, interrogation totale donc; et celle en vue d'obtenir une information sur les circonstances ou sur l'objet, type de la question partielle. Afin de montrer quel type de formulation appelle la visée de la question, une dimension comparative avec d'autres variétés de français parlé m'est apparue pertinente. J'ai donc regardé aussi les faits ou leurs représentations ${ }^{4}$ dans d'autres variétés nord-américaines, ainsi que dans «le français parlé de France $»^{5}$. La perspective comparative cherche à mettre en évidence des divergences et des convergences entre ces divers français parlés.

Le relevé n'est pas statistique, et mon approche sera qualitative : les possibilités d'apparition d'une occurrence en syntaxe étant toujours plus faibles que pour des domaines comme la phonologie, la fréquence « jouera donc un moindre rôle [en outre] ce n'est pas sa fréquence qui rendra une forme syntaxique saillante » (Gadet 1997b, p. 16).

\footnotetext{
${ }^{4}$ Les faits n'étant pas toujours, surtout pour le français de France, établis d'après des relevés de corpus mais à partir d'énoncés fabriqués.

${ }^{5}$ Cette appellation permet une dénomination unique de ce que d'aucuns appellent français populaire, français avancé ou français ordinaire.
} 


\section{Relevé et analyse}

Je me concentrerai donc sur la demande d'information (la demande de dire), réduite ici à trois visées: réponse totale (oui / non), sur les circonstances, ou sur l'objet.

\subsection{L'interrogation appelle une réponse "fermée » (oui /non)}

Lorsque la question est une interrogative totale appelant une réponse affirmative ou négative, l'interrogation passe par divers moyens, y compris ceux signalés comme rares dans d'autres variétés.

\section{- L'inversion}

L'inversion simple est bien illustrée dans le corpus :

pis payais/ payais-tu pour rester là (St. NB : 55-56)

étiez-vous comme une famille qu'était proche ou c'était-ti (Ch. NB : 4-5)

Sa présence chez plusieurs locuteurs témoigne de son usage courant. L'inversion interrogative étant essentiellement relevée à la deuxième personne du singulier, il y a sans doute un lien avec les particules postposées tu et ti. Mais, étant donné le genre de paroles recueillies, la majorité des interrogatives relevées sont justement à la deuxième personne du singulier. Il est donc difficile de trancher. Quoi qu'il en soit, l'inversion simple fut fréquemment relevée dans des corpus de français acadien traditionnel $^{6}$ (Melkersson, 1979, p. 171 et Gesner, 1984-1985, p. 131). On trouve cette structure à une fréquence plus élevée qu'en France dans d'autres variétés canadiennes (Rodriguez, 1991, p. 168-169 pour le français manitobain).

L'interrogation par inversion complexe fait partie du répertoire dans des contextes plus formels comme l'écrit.

Mais pépère, toi et ta génération ... où aviez-vous la tête ? ( $A N, 02.07)$

Jacques Chirac vient-il chercher son inspiration en Acadie ? ( $A N, 02.07)$

Pourquoi la police rend-elle la vie dure aux amateurs de VTT ? (AN, 02.07)

Sa rareté dans les prises de parole orales en Acadie ne nous apprend rien de spécifique sur la variété.

- L'introducteur est-ce que

est-ce que le service est mieux. je le sais pas (R. NB : 197)

pis::: est-ce que/ est-ce que tu voudrais rester dans cette région là pour/ pour le reste de ta vie ou est-ce que (Mt. NB : 5-6)

ta mère est-ce qu'elle travaillait pendant sa jeunesse (Ch. NB : 19-20)

\footnotetext{
${ }^{6}$ On entend par là l'état de langue reflété dans des corpus constitués avant l'émancipation de la communauté acadienne (avant les années 1960), recueillis surtout en milieu rural auprès de témoins âgés.
} 
Ce groupe est considéré comme très fréquent en France (Riegel et al. : 393, voir aussi Blanche-Benveniste et al 2002). Cette construction relativement bien représentée dans mon corpus au Nouveau-Brunswick est réputée absente des corpus les plus anciens (Gesner 1984-1985). Elle apparaît dans le corpus néo-brunswickois de Wiesmath, comme dans le mien. Wiesmath suggère une influence récente du français de France (2006, p. 182).

\subsection{La question constitue une demande d'informations (sur les circonstances)}

Lorsque la question est une interrogative partielle sur les circonstances, est-ce que est très fréquent. Il est la plupart du temps précédé d'un adverbe interrogatif, y compris à l'écrit.

Comment est-ce qu'un être humain en parfaite santé peut vouloir subir une intervention chirurgicale pour changer son apparence ( $A N, 02.07$ notons que le propos se donne comme une citation)

Les groupes interrogatifs formés d'un adverbe interrogatif suivi de est-ce que sont assez anciens en français acadien, signalés par Poirier. La forme adverbe interrogatif + est-ce que se rencontre dans nombre de questions partielles portant sur les circonstances.

Ce sont ces dernières - qualifiées en français parlé de France de «familières » ou «populaires» (Riegel et al., p. 398-399) - qui vont maintenant nous retenir. Certaines sont si répandues que les grammaires du français leur accordent parfois une place. J'ai donc choisi d'étudier les convergences et les divergences. Nous allons voir que dans le corpus s'illustre une tendance qui met en évidence le terme interrogatif ou la locution interrogative. Cette mise en évidence d'un élément sémantiquement explicite (où pour interroger sur le lieu, pourquoi pour les causes,...) se retrouve dans d'autres variétés de français parlés. En revanche, les modalités de mise en évidence de l'élément peuvent varier. Dans le corpus, c'est la position en tête d'énoncé qui domine. On relève en effet diverses stratégies d'extraction des termes ou des locutions interrogatives.

En français parlé de France, l'interrogation avec terme interrogatif occupant la place du constituant, comme dans Tu attends qui ? Tu regardes quoi? Tu vas où? Tu pars quand? est réputée très fréquente (Riegel et al., p. 398), s'appliquant à toutes les fonctions sauf le sujet. Mais elle est absente de mon corpus, et n'est pas recensée dans les travaux sur la variété. Je ne l'ai pas trouvée mentionnée non plus dans les travaux sur le français québécois (Léard, 1995) ou sur d'autres variétés du Canada (Hallion, 2006 pour l'Ouest). De fait, dans le corpus pour les interrogations sur les circonstances, les structures à terme interrogatif en première position dominent.

Plus largement, si les stratégies d'extraction d'un terme interrogatif pour interroger sur les circonstances sont un point commun des français parlés de France et d'Acadie, les modalités de mise en évidence peuvent diverger. C'est surtout la place de l'élément interrogatif qui diffère. Ainsi du présentatif c'est que associé à un terme interrogatif, stratégie d'extraction d'un terme interrogatif fréquente en français parlé en France (Riegel et al: 398). Le terme interrogatif peut précéder c'est que, ou être inséré 
dans le présentatif pour des constructions de type c'est + terme interrogatif + que (c'est quand que, c'est ò̀ que). Dans mon corpus, les structures dans lesquelles le terme interrogatif est en première position suivi de c'est que dominent, pour le lieu (où c'est que), la cause (pourquoi c'est que) ou d'autres circonstances.

où c'est que t'as voyagé dans ta vie (Ch. NB:171)

pis y en a un qui m'avait demandé pourquoi c'est que je restais à la même JOB tout le temps . pour vingt ans (Z. NB : 480-482)

La préférence est toujours donnée à la structure qui fait précéder le terme interrogatif, et certaines formes pourraient être en cours de lexicalisation. La fréquence d'apparition du verbe être est en effet remarquable ${ }^{7}$, en des formulations qui peuvent être estimées lexicalisées. Au rang des plus courantes, comment-ce que :

l'Université de Moncton comment-ce que tu trouves ça parle moi de ton arrivée à l'Université de Moncton (Mt. NB : 21-22)

comment-ce qu'i s'appelle (Z. NB : 297)

on l-avait un:: comment-ce que t'appellerais ça un rouleau (Ai. NB : 149)

comment-ce qu'elle a fait appeler son bébé MAM (Ca. NB : 39-40)

après la viande est cuite on faisait dans: . comment-ce qu'on dit ça un:/ un:/ un:/ je sais pas (Ed. NE 26-27)

Pour le même type de construction, où-ce que a acquis le statut de véritable locution. Il est attesté en France, où il est qualifié de « franchement populaire » (BU \ 390-b) et signalé dans plusieurs français d'Amérique (au Québec notamment, Léard 1995).

ben oùsqu'elle/ qu'elle a pris son cours d'éducation sexuelle elle là (Sl. NB : 273)

Pour interroger sur l'objet, nous retrouvons une structure similaire avec quoi-ce que. Toujours pour les circonstances, en mettant en avant le terme interrogatif, on trouve des interrogatifs composés qui élident le groupe complet [démonstratif + verbe etre] : pourquoi que, comment que, combien que, voire quoi que pour l'interrogation sur l'objet (voir plus bas).

WELL vont dire pourquoi que t'as acheté ça pour (Z. NB : 195)

pourquoi que vous l'avez pris chez vous (G. IPE : 132)

où est-ce que y a/ la/ la sève là $[\ldots]$ comment que t'appelles (St. NB : 383)

comment que tu te sentais comme en disant t'es français (Sf. IPE: 312-313)

combien qu'i payont . je crois trois piasses et demi (W. NB : 45)

Certaines de ces formes ont une assise historique : comment que et combien que sont toutes deux attestées anciennement et notées de longue date dans le français acadien (Gérin et Gérin, 1979, p. 103). En l'état actuel de la langue, il n'est pas évident de savoir si nous devons considérer la présence de que derrière des termes interrogatifs comme une conservation de formes anciennes, comme un ajout à un terme interrogatif, ou comme le reliquat de la forme étoffée : terme interrogatif + est-ce / c'est + que. Sa présence peut s'expliquer aussi par la perception de que comme élément de

${ }^{7}$ La différence phonique entre comment est-ce que et comment ce-que est parfois difficile à saisir. 
subordination par excellence (Arrighi, 2005), l’interrogation partielle étant un énoncé subordonné. Dans le français acadien tel que reflété notamment par le corpus Wiesmath ou par le mien, que peut toujours s'adjoindre à un élément de relation, un relatif (où que, qui que), une conjonction (si que, quand que) et à diverses constructions (malgré que). Ce type de construction n'est d'ailleurs pas spécifique au français parlé en Acadie. Pour les seules interrogatives, les formulations de type terme interrogatif + que se trouvent dans le «français populaire » (Guiraud 1969, p. 73) et de façon générale dans les français d'Amérique ${ }^{8}$.

La présence de que dans l'interrogative - même s'il ne s'insère pas dans une conjonction complexe - est très fréquente, que ce soit avec des adverbes interrogatifs ou avec l'adjectif quel.

combien de cartes que vous jouez (G. IPE : 10-11)

combien d'élèves que y avait à peu près dans chaque classe (Sf. IPE: 45-46)

quel âge qu'elle a Véronique (Ct. NB : 177)

quel âge qu'elle avait (S. NB: 68)

à quelle place qu'i restait (G. IPE : 3)

à quelle école que t'allais (Ch. NB : 16)

ben à quelle place qu'i reste (Cr. NB : 299)

Les exemples ci-dessus, s'ils se ressemblent, ne sont peut-être pas à analyser de la même façon. Dans les constructions adverbe interrogatif + nom + que, il n'est pas évident que que joue un rôle autre que marquer la thématisation de l'objet. La formulation avec adjectif interrogatif quel + nom + que, sans doute à mettre en parallèle avec la propension à utiliser que dans l'interrogative, est l'usage le plus commun pour un adjectif interrogatif. Les structures sans que sont en effet absentes du corpus. Cette forme est possible en français parlé de France, où elle est jugée «très populaire », et elle est relevée ailleurs au Canada 9 . Il n'est pas évident de considérer ce que comme simple explétif, sans doute faut-il lui accorder un statut de complémenteur. Si que joue un rôle important dans les interrogatives sur les circonstances (qui peuvent être considérées comme un type particulier de circonstancielles), sa présence est plus erratique dans les interrogatives sur l'objet (qui peuvent être regardées comme un type particulier de complétives).

\subsection{La question constitue une demande d'informations (sur l'objet)}

Comme signalé ci-dessus, on trouve des locutions construites avec un terme interrogatif (massivement quoi) de forme identique aux locutions pour les circonstances (terme interrogatif $+c e+q u e$, ou terme interrogatif $+q u e$, ici quoi-ce que et quoi que). Quoi-ce que est courant pour l'objet:

\footnotetext{
${ }^{8}$ Papen relève combien que dans le français des Métis manitobains (2002, p. 127), Chaudenson et al. en français louisianais (1993, p. 117).

${ }^{9}$ Papen (1998, p. 158).
} 
Laurence Arrighi

pis quoi-ce que vous faisiez sus vos soirées (St. NB : 71-72)

oùs t'as pris ça . quoi-ce que c'est (Cr. NB : 323)

mais dit quoi-ce que tu vas faire icitte (M. NE : 416-418)

Cette forme se trouve dans d'autres variétés nord-américaines ${ }^{10}$, de même que quoi que.

quoi que tu fais icitte (M. NE: 111)

quoi que tu vas porter à papa (M. NE : 147)

Mais l'observation des modalités de l'interrogation, quand l'interrogative porte sur l'objet,_laisse apparaittre des constructions sans complémenteur que:

vous allez voir au fond quoi c'est là (M. NE : 353)

le dernier . des garçons i l'ont fait a/ appeler Roch ben quoi ça veut dire ça en français Roch (Rt. NB: 44-46)

pis la polyvalente LJR qu'est-ce * t'en penses (Mt. NB : 80)

qu'est-ce * c'était ta matière préférée $(\mathrm{Ch} . \mathrm{NB}: 25)$

Quoi-ce (équivalent de «qu'est-ce, qu'est-ce que » ?) se trouve dans divers français nord-américains ${ }^{11}$ et dans le français parlé de France notamment avec des formes incluant c'est, ce ou ça. Il n'est pas sûr pour autant que ces formes disposent d'assises historiques. Pour l'ensemble des formes incluant c'est, ce, ça, Chaudenson et al. avancent que: «[o]n peut admettre que l'ensemble de ces emplois témoigne aussi de la recherche de «l'expressivité » dont parle Frei » $(1993: 115)^{12}$. Au minimum, il parait pertinent de les mettre en relation avec les autres tournures à présentatif.

Remarquons en particulier, pour l'économie de que élément de conjonction, ce qui est illustré par les deux derniers exemples. Cette forme me semble courante en France au moins chez les jeunes générations. Parce qu'elle ne fait pas vraiment partie de la variété qu'exporte l'ancienne métropole, on peut conclure à deux évolutions autonomes. La présence de deux tendances identiques dans les français parlés en France et en Acadie, présence importante de que dans divers types de constructions pour les interrogatives sur les circonstances, et ellipse fréquente quand l'interrogative porte sur l'objet, incite à replacer les faits dans le contexte de la subordination. La généralisation de que conjonctif pour les circonstancielles et son ellipse fréquente pour les complétives (Arrighi, 2005, p. 280-303; Wiesmath, 2006, p. 177-181) montre que l'étude des interrogatives gagne à être insérée dans une perspective plus large d'observation de construction des énoncés à l'oral.

Cette mise en contexte interne des productions parlées, ainsi qu'un traitement de la question selon une perspective plus linguistique (visées pragmatico-sémantiques, contraintes syntaxiques) semblent plus productifs que le questionnement sociologique : la variation en syntaxe ne constituerait ainsi pas un lieu privilégié « d'interface

\footnotetext{
${ }^{10}$ Voir notamment Stäbler $1995: 165$ pour le français cadien ; King et Butler $(2005$, p. 176) pour le franco-terreneuvien.

${ }^{11}$ En français acadien de Louisiane et en français louisianais (Chaudenson et al. 1993, p. 114 et 113).

12 Guiraud recueille les formes incluant c'est, ce dans «la série des emphatiques » (1969, p. 49).
} 
entre le linguistique et le social»(Gadet, 1997a, p. 17). Nous sortons ainsi d'une linguistique descriptive qui traite les variétés non standardisées selon une problématique de registre de langue et non comme un champ d'observation fécond. Si l'on ajoute que pour le moment nous n'avons encore qu'une « connaissance imparfaite » de la «structure syntaxique et discursive de la langue telle qu'elle est vraiment parlée » (Gadet, 1997a, p. 17), il ne me semble faire aucun doute que l'étude des français parlés hors de France puisse apporter des éclairages profitables à la description du français parlé tout court.

Cette approche de la variabilité des formes en syntaxe éloignée des contingences sociales a permis de mettre au jour le fait que si les interrogatives en français parlé présentent une multiplicité de formes, le choix d'une structure interrogative n'est pas absolument dépendant du profil du locuteur; de même qu'il n'est pas parfaitement libre. La recherche d'une adéquation entre formes de la question et contraintes linguistiques internes fait apparaitre des tendances (car il est toujours difficile d'établir des lois qui constitueraient les règles infaillibles d'une grammaire de l'oral). Ces tendances montrent que les procédés semblent, au moins en partie, dépendre du type d'interrogation. Enfin, à un autre niveau, la dimension comparative de cette étude très ponctuelle fait émerger certaines convergences de fonctionnement entre français parlés en divers lieux géographiques. Ceci amène aussi à considérer avec précaution la notion de variété géographique de langue, pour ce qui a trait à la syntaxe du français parlé pour le moins.

\section{RÉFÉRENCES BIBLIOGRAPHIQUES}

ARRIGHI, L., (2005), Etude morphosyntaxique du français parlé en Acadie - Une approche de la variation et du changement linguistiques en France, thèse de doctorat, Université d'Avignon.

BARbArIE, Y., (1982), «Analyse sociolinguistique de la syntaxe de l'interrogation en français québécois » Revue québécoise de linguistique, 12-1, p. 145-167.

Blanche-Benveniste, C., (1997), «La notion de variation syntaxique dans la langue parlée » Langue française, 115 , p. 19-29.

--- et al. (éds) (2002), Choix de textes de français parlé - 36 extraits, Paris, Honoré Champion.

COVEnEY, A., (1995), «The use of the QU-final interrogative structure in spoken French» Journal of French language Studies, 5, p. 143-171.

--- (1996), Variability in Spoken French. A sociolinguistic Study of Interrogation and Negation, Exeter, Elm Bank.

--- (1997), «L'approche variationiste et la description de la grammaire du français : le cas des interrogations » Langue française, 115, p. 88-100.

Chaudenson, R., Mougeon, R et Beniak, E., (1993) Vers une approche panlectale de la variation en francais, Didier-Erudition, Paris. 
Laurence Arrighi

GADET, F. (1997a), Le français ordinaire, Colin, Paris.

--- (1997b), «La variation, plus qu'une écume » in Langue française, 115, p. 5-18.

Gerin, P., et GERIN, P-M. (1979), «Eléments de la morphologie d'un parler franco-acadien : remarques sur la langue de Marichette», Si que, 4, p. 79-110.

GeSNER, E. (1984-85), «Les structures interrogatives dans le parler acadien de Pubnico (NouvelleÉcosse) », Journal of the Atlantic Provinces Linguistic Association, 6-7, p. 124-171.

Guiraud, P. (1969), Le français populaire, Paris, PUF.

HALLION, S. (2006), «Similarités morphosyntaxiques des parlers français de l'Ouest canadien » Revue canadienne de linguistique appliquée, 9, 2 / Revue de l'Université de Moncton, 37, 2, p. 111-131.

LÉARD, J-M. (1995), Grammaire québécoise d'aujourd'bui, Guérin Universitaire, Montréal.

King, R., et Butler, G. (2005), «Les Franco-Terreneuviens et le franco-terreneuvien » in Valdman, Auger et Piston-Hatlen (dir.) Le français en Amérique du Nord - Etat Présent, PUL, Québec, p. 169-185.

PAPEN, R. (1998), «Le parler français des Métis de l'Ouest canadien » in Brasseur (éd.) Français d'Amérique - variation, créolisation - normalisation, Centre d'Études Canadiennes, Avignon, p. 147-161.

--- (2002), «Quelques remarques sur un parler français méconnu de l'Ouest canadien : le Métis » Revue québécoise de linguistique, 14, 1, p. 113-139.

PÉRONNET, L. (1993) «La situation du français en Acadie : de la survivance à la lutte ouverte » in de Robillard \& Beniamino (éds.) Le français dans l'espace francophone, Champion, Paris, p. 102-116.

PoIrIER, P. (1993), Le Glossaire Acadien édition critique établie par Gérin, Centre d’Études Acadiennes - Université de Moncton, Moncton, Les Éditions d'Acadie.

Quillard, V. (2001), « La diversité des formes interrogatives », Langage et société, 95, p. 57-72.

RIEGEL, M., et al. (1996), Grammaire méthodique du français, PUF, Paris.

RoDRIGUEZ, L. (1991), « Aspects morphologiques et syntaxiques du parler franco-manitobain », in Horiot, Français du Canada-Français de France, Niemeyer, Tübingen, p. 161-172.

STÄBLER, C. (1995), Entwicklung mündlicher romanischer Syntax: das français cadien in Lonisiana, Tübingen, Narr.

WIESMATH, R. (2000), Enchaînement des propositions dans le français acadien du Nouveau-brunswick/ Canada, thèse de doctorat, Albert-LudwigUniversität Freiburg.

--- (2006), Le français acadien - Analyse syntaxique d'un corpus oral recueilli au Nouveau-Brunswick / Canada, L'Harmattan, Paris. 